\title{
Die Genderperspektive als Modernisierungsstrategie: Frauen in pädagogischen Führungspositionen im transatlantischen Vergleich
}

\section{Claudia Fahrenwald und Maureen Porter}

Im Rabmen der aktuellen gesellschaftlichen Modernisierungsprozesse ist auch die Genderperspektive auf eine neue Weise in das Blickfeld sozialwissenschaftlicher wie politischer Diskussionen gerïckt. So wurde durch den Amsterdamer Vertrag von 1998 die Umsetzung von Gender Mainstreaming, und damit die Gleichstellung von Männern und Frauen in allen Lebensbereichen, zur offiziellen Richtlinie europäischer Politik (Bergmann \& Pimminger 2004). In Deutschland sind jedoch in allen wichtigen gesellschaftlichen Bereichen Frauen in Führungspositionen nach wie vor deutlich unterrepräsentiert. Dies gilt auch für den Bereich der Schule als einem für die zukünftige gesellschaftliche Entwicklung besonders sensiblen Handlungsfeld. Es erscheint daher sinnvoll und von allgemeinem gesellschaftlichem Interesse, die Situation von Frauen in pädagogischen Führungspositionen einmal vor dem Hintergrund der traditionellen Strukturen im Schulwesen zu erfassen und auf diese Weise den Blick auf eine von der Führungsforschung bislang eher "wenig beachtete Teilgruppe innerhalb der gerne beleuchteten Gruppe der Führungsfrauen» (KansteinerSchänzlin 2004, S. 7) zu lenken. Was hindert Frauen daran, eine pädagogische Führungsposition anzustreben? Und welche Erfahrungen haben Frauen auf ihrem Weg in eine pädagogische Führungsposition gemacht? Diese Fragen stehen im Mittelpunkt des folgenden Beitrags, der zu diesem Thema ausgewählte Ergebnisse eines transatlantischen Forschungsprojekts präsentiert. In den angloamerikanischen Ländern besitzt die Diskussion der "Qualities of Leadership" bereits eine lange Tradition. Ein Aufgreifen dieser Thematik in einem internationalen Rahmen ermöglichte daher interessante Perspektiven der kulturvergleichenden Analyse und Kooperation.

Mit dem Amsterdamer Vertrag von 1998 und dem damit verbundenen Beschluss zur Umsetzung von Gender Mainstreaming wurde die Gleichstellung von Männern und Frauen in allen Lebensbereichen zur offiziellen Richtlinie europäischer Politik (Bergmann \& Pimminger, 2004). Dennoch sind in vielen europäischen Ländern Frauen in Führungspositionen in allen wichtigen gesellschaftlichen Bereichen wie der Wirtschaft, der Wissenschaft und der Politik nach 
wie vor deutlich unterrepräsentiert. Dies gilt in Deutschland auch für den Bereich der Schule als einem für die zukünftige gesellschaftliche Entwicklung besonders sensiblen Handlungsfeld. Gerade im Bildungsbereich geraten jedoch seit einigen Jahren überkommene Organisationsstrukturen und Handlungsroutinen zunehmend unter Druck. Damit bietet sich die Chance, auch auf die Modernisierungsrückstände bei der Teilhabe der Geschlechter an pädagogischen Führungspositionen hinzuweisen. Geht man in diesem Zusammenhang davon aus, dass Internationalisierungsprozesse einen wichtigen Auslöser für gesellschaftliche Restrukturierungsprozesse darstellen, so erscheint es hier sinnvoll und von allgemeinem gesellschaftlichen Interesse, einmal einen Blick über die europäischen Grenzen hinaus zu werfen.

Ein etwas anderes Bild präsentiert sich bei diesem Thema in den USA, wo die Diskussion um Gender Equitiy bereits auf eine längere Tradition zurückblicken kann. Zwar sind auch im amerikanischen Schulsystem mehr Männer als Frauen in Führungspositionen vertreten, doch stellen sich die Karrierechancen für Frauen im Schulbetrieb dort insgesamt deutlich günstiger dar. Anders als in Deutschland ist z.B. ein flexibler Aufstieg aus dem Schuldienst bis in höchste Positionen der Schulverwaltung sowie ein Wechsel in die universitäre Laufbahn möglich. Prozentual befinden sich in den USA daher deutlich mehr Frauen in einer leitenden schulischen Funktion. Allerdings bleiben sie auch dort oftmals trotz hervorragender fachlicher Qualifikation in einer nachgeordneten Stellvertreterposition stecken (Hodgkinson \& Montenegro, 1999). Die Forderung nach einer Erhöhung des Frauenanteils in pädagogischen Führungspositionen stellt daher auch in der amerikanischen Schulentwicklungsdebatte ein wichtiges Thema dar (Bastian \& Greer, 1985; Shakeshaft, 1993; Sobehart, 2008).

Vor diesem Hintergrund fand in den Jahren 2000-2002 parallel an den Universitäten Augsburg (Bayern) und Pittsburgh (Western Pennsylvania) ein transatlantisches Forschungsprojekt über Frauen in pädagogischen Führungspositionen statt. Ziel des Projekts war es, aus einer kulturvergleichenden Perspektive die Aufmerksamkeit auf eine von der sozialwissenschaftlichen Führungsforschung bislang eher "wenig beachtete Teilgruppe innerhalb der gerne beleuchteten Gruppe der Führungsfrauen» (Kansteiner-Schänzlin, 2004, S. 7) zu lenken. Ausgangspunkt des Projekts bildeten folgende Fragestellungen: Welche Chancen, aber auch welche Probleme sehen Frauen in Deutschland und in den USA, wenn sie sich für eine pädagogische Führungsposition bewerben? Und welche Erfahrungen haben Frauen in beiden Ländern auf ihrem Weg in eine pädagogische Führungsposition gemacht? Mit ausgewählten Ergebnissen des Projekts beschäftigt sich der folgende Beitrag und diskutiert diese insbesondere im Hinblick auf ihr modernisierungsthoretisches Potenzial für die Ausgestaltung einer geschlechtergerechten Schulkultur für die Schule von morgen. 


\section{Schule im Kontext gesellschaftlicher Modernisierungsprozesse}

Die Einsicht, dass Schule sich verändern müsse, um dem raschen gesellschaftlichen Wandel zu begegnen, hat sich mittlerweile in allen westlichen Industrienationen durchgesetzt. Als Auslöser für umfassende Reformdiskussionen können hier für Europa die Ergebnisse der PISA-Studie aus dem Jahr 2000 (http://www.mpib-berlin.mpg.de/pisa) und für die USA die «No Child Left Behind»-Initiative von 2001 (http://www.ed.gov/nclb/landing.jhtml) angesehen werden. Erste Reformansätze finden sich mittlerweile im Bereich der Lerninhalte und Lernformen sowie bei der Einführung neuer Schultypen oder Ausbildungszweige. Weitgehend unangetastet erscheinen dagegen oftmals die inneren Strukturen des Schulwesens selbst. Hier hat sich im Rahmen der aktuellen Schulentwicklungsdebatte in den vergangenen Jahren die Aufmerksamkeit für die Person des Schulleiters deutlich erhöht, da ihr im schulinternen Reformprozess eine zentrale Rolle zugeschrieben wird. In diesem Zusammenhang geriet auch das traditionelle «patriarchalische» Bild des Schulleiters zunehmend in die Kritik, da es angesichts immer komplexerer Aufgabenstellungen sowie eines permanent steigenden Verantwortungsdrucks nicht mehr zeitgemäß erschien und durch ein teamorientiertes Konzept von «Führung» ersetzt werden sollte (Chott \& Schmirber, 2003; Rosenbusch, 1997).

In diesem Zusammenhang springt auch eine Tatsache ins Auge, die auf die mangelnde Gendersensibilität des traditionellen Schulsystems verweist: Obwohl der Lehrerberuf als ein typischer «Frauenberuf» gilt (Glumpler, 1993), ist der Anteil von Frauen in Führungspositionen in Schulen und Schulbehörden in Deutschland nach wie vor vergleichsweise gering. Dies gilt vor allem für den Bereich des Gymnasiums als einem besonders prestigeträchtigen Schultypus. Zwar wird hier die niedrige Quote an weiblichen Schulleitungen unter Hinweis auf entsprechende Statistiken bereits seit Mitte der 1980er Jahre kritisiert, doch hat sich an der Situation bis in die 1990er Jahre hinein wenig verändert (Biermann \& Koch-Priewe, 2004). Das Berufsziel «Schulleitung» erscheint für Frauen in der Regel offensichtlich sehr viel weniger selbstverständlich als für ihre männlichen Kollegen (Kreienbaum, 1995). Empirische Untersuchungen bescheinigten in der Vergangenheit Lehrerinnen auch oftmals nur ein geringes Karriereinteresse, das diese mit ihrer unmittelbaren Ausrichtung auf die pädagogische Arbeit sowie mit der Befürchtung begründeten, durch den Aufstieg in eine berufliche Führungsposition persönliche Kontakte zu Kolleginnen und Kollegen zu gefährden (Brehmer, 1992; Flaake, 1989). Zudem äußerten viele Frauen eine prinzipielle Distanz gegenüber «männlichen» Formen und Inhalten der Arbeit in schulischen Gremien oder Funktionsstellen (Koch-Priewe, 1996) sowie eine kritische Einstellung zum Thema Macht (von Lutzau, 1996). 


\section{Frauen in pädagogischen Führungspositionen: Ein transatlantischer Vergleich}

Ausgehend von diesem Forschungsstand wurde das transatlantische Forschungsprojekt «Frauen machen Schule» über Frauen in pädagogischen Führungspositionen durchgeführt (Fahrenwald \& Porter, 2002), das sich an Frauen in einer pädagogischen Führungsposition oder mit einem prinzipiellen Interesse an einer pädagogischen Führungsposition richtete. Die empirische Untersuchung fand an ausgewählten Gymnasien in der Region Schwaben (Bayern) und an vergleichbaren Schulen (Middle Schools und High Schools) in Western-Pennsylvania (USA) statt. Die Rekrutierung des Samples erfolgte mittels einer Verbindung von quantitativen und qualitativen Erhebungsmethoden: Zunächst wurde mit Hilfe eines Fragebogens eine erste Kontaktaufnahme mit den für die Untersuchung in Frage kommenden Frauen hergestellt. Anschließend wurde mit einzelnen Gesprächspartnerinnen ein biographisch orientiertes, leitfadengestütztes Interview durchgeführt, in dem diese über ihren persönlichen Werdegang sowie über ihre Erfahrungen mit dem Thema «Führungspositionen» berichteten.

In Pittsburgh finden im Rahmen einer an die Universität angegliederten Leadership Academy bereits seit Jahren regelmäßig Weiterbildungen für Lehrerinnen in oder mit einem prinzipiellen Interesse an einer pädagogischen Führungspositionen statt, wo diese das in den USA staatlich vorgeschriebene Zertifikat für Führungskräfte erwerben können (Curry, 2000). In den USA war es daher kein Problem, geeignete Frauen für die Untersuchung zu finden. Dazu wurde an alle interessierten Teilnehmerinnen der Weiterbildungsveranstaltungen ein gemeinsam entwickelter Fragebogen verteilt, in dem statistische Sozialdaten, allgemeine Einstellungen zu den Themen «Führungspositionen» und «Macht» sowie die grundsätzliche Bereitschaft zur Teilnahme an einem Interview abgefragt wurden. In Deutschland gestaltete sich die Suche nach geeigneten Interviewpartnerinnen dagegen deutlich schwieriger, da es zum damaligen Untersuchungszeitpunkt im definierten Untersuchungsgebiet nur insgesamt drei (!) Schulleiterinnen an staatlichen Gymnasien gab und gleichzeitig ein offizielles Forum für Austausch und Begegnung fehlte. Es wurden daher an alle 55 Gymnasien in Schwaben insgesamt 150 Fragebögen verschickt, die sich jeweils mit einem kurzen Begleitschreiben an die Schulleitung des Gymnasiums richteten und mit der Bitte um eine Weiterleitung der Fragebögen an aus Sicht der Schulleitung geeignete, bzw. interessierte Lehrerinnen versehen waren. Eine Rücklaufquote von $53 \%$ kann hier als ein sehr positives Ergebnis angesehen werden. Offensichtlich bestand ein aktuelles Interesse am Thema.

Am Ende des Fragebogens wurde auch in der deutschen Untersuchungsgruppe nach der Bereitschaft gefragt, an einem weitergehenden Interview teilzunehmen. Die Anzahl der an einem Interview interessierten Lehrerinnen überstieg in beiden Ländern deutlich die für die Untersuchung vorgesehene Anzahl an Interviews. Es musste daher eine Auswahl der Interviewpartnerinnen getrof- 
fen werden. Dabei wurde sowohl für das deutsche als auch für das amerikanische Sample auf eine möglichst gleichmäßige Repräsentation von städtischen und ländlichen Schulen, von jüngeren und älteren Kolleginnen sowie von Frauen mit, bzw. ohne Kinder geachtet. Auf diese Weise wurden schließlich im Rahmen des Projekts jeweils 20 Frauen in beiden Ländern nach ihrem beruflichen Werdegang und ihrer Einstellung gegenüber einer schulischen Führungsposition befragt. Das Untersuchungsinteresse galt dabei den institutionellen Rahmenbedingungen für einen beruflichen Aufstieg sowie den persönlichen Erwartungen, Erfahrungen und Problemen der Frauen selbst. Die Interviews wurden auf Tonträger aufgenommen und anschließend mit Hilfe einer computergestützten Analyse im Hinblick auf vorher gemeinsam definierte Kategorien ausgewertet. Im Mittelpunkt standen dabei folgende Themen:

- die individuellen beruflichen Karrierewege der Frauen,

- ihre Einstellungen zu den Themen «Führung» und «Macht»,

- ihr Bedarf an zukünftigen Weiterbildungsangeboten.

Im Folgenden werden einzelne Untersuchungsergebnisse näher vorgestellt.

\section{Ambivalenzen des beruflichen Aufstiegs}

Als ein zentrales Ergebnis der Studie kann die Tatsache angesehen werden, dass viele der befragten Frauen einem beruflichen Aufstieg prinzipiell ambivalent gegenüber standen (Fahrenwald \& Porter, 2006). Dieses Ambivalenzgefühl wurde zum Teil durch individuelle Ambivalenzerfahrungen im Verlauf der persönlichen beruflichen Entwicklung bestätigt. So distanzierte sich die Mehrzahl der befragten Lehrerinnen in der deutschen Untersuchungsgruppe von Anfang an ausdrücklich von der mit dem Begriff «Aufstieg» verbundenen Assoziation eines «sich Erhebens» über andere und betonte den Aspekt eines «inneren Vorwärtskommens». Die beiden folgenden Aussagen verdeutlichen diese Einstellung exemplarisch:

Vorwärtskommen. Ja. Also, Aufstieg würde mir nicht behagen, das würde wieder bedeuten - jetzt mal ganz symbolisch gesehen - ich steige über die anderen und sehe dann wieder jemanden von oben, und das wäre mir nicht wichtig, über jemandem zu stehen (D Interview 13: 294).

Also, ich sehe es für mich jetzt mehr idealistisch, auch als Persönlichkeitsreifung (D Interview 17: 238).

Dieses Ergebnis wird von vorhergehenden Studien bestätigt, in denen ebenfalls deutlich wurde, dass sich die Aufstiegsmotivation von Lehrerinnen in der Regel von der ihrer männlichen Kollegen unterscheidet: Für Frauen stehen im Hin- 
blick auf eine Führungsposition mehrheitlich nicht materielle, ökonomische oder macht- und prestigebezogene Gründe im Vordergrund, sondern persönliche Motive und ideelle Werte, wie z.B. neue Herausforderungen oder Gestaltungsmöglichkeiten (Werle, 2001). Ähnliche Tendenzen lassen sich auch im amerikanischen Sample der aktuellen Studie feststellen, wo immer wieder der mit einer Führungsposition verbundene Zugewinn an Handlungsspielräumen betont wurde:

I don't know about upward, but it was a forward move (...) I like the idea of forward. I like the term forward. Being able to move the goal, the organization (...) Leadership is so dynamic! Leadership is the ability to be effective in moving people toward a goal (US Interview 1: 449-455).

Insgesamt zeichnete sich die amerikanische Untersuchungsgruppe durch eine größere Aufgeschlossenheit gegenüber der Führungsthematik aus, was möglicherweise mit der langen Tradition der Ausbildung von "Qualities of Leadership» in den angloamerikanischen Ländern zu erklären ist. Dennoch wurden auch von den amerikanischen Interviewpartnerinnen ihre Karrierewege keineswegs als unproblematisch dargestellt, sondern waren gekennzeichnet durch Brüche und Ambivalenzen.

\section{Metaphern der Dunkelheit}

So schilderten auch diejenigen der amerikanischen Lehrerinnen, die bereits auf einen erfolgreichen beruflichen Werdegang zurückblicken konnten, diesen im persönlichen Rückblick häufig als einen mühevollen Weg voller Unwägbarkeiten und Turbulenzen. Auffällig waren dabei insbesondere Metaphern der Dunkelheit, mit denen sie ihre beruflichen Erfahrungen umschrieben. Es war die Rede vom Überwechseln zur «dark side», vom Durchqueren eines «dark tunnel» oder vom Eingeschlossensein in einem «dark cave»:

My career path has been like a ride on a wonderful roller coaster. There have been ups and downs. There have been times when you just squeal and laugh and celebrate and have great joy. There have been other times where you just go into those dark tunnels and you are like: "What am I doing and why am I doing this?" (US Interview 2: 48) (Herv. v. d. Verf.).

In einem der Interviews wurde bereits die Entscheidung für eine Führungsposition als ein «Tappen im Dunkeln» beschrieben, als eine unübersichtliche Situation voller Angst vor möglichen Verlusten:

Teacher: I think of it as going into a dark cave and you're not sure whether there is going to be a light at the end of it (...)

Interviewer: Okay. Where would the light come from? 
Teacher: From if you've done the right thing for you or not.

Interviewer: What's the dark cave?

Teacher: Just making that transition, deciding to give up your classroom, and then going into something completely different. And it can also be making that transition to another school district - yeah, I think that would be very difficult. (US Interview 11: 567-575) (Herv. v.d. Verf.).

Der Aufstieg in eine Führungsposition wird somit von vielen Frauen keineswegs als eine eindeutige Erfolgsgeschichte angesehen, sondern als ein komplexer und komplizierter biographischer Balanceakt, der unmittelbar in subjektive Handlungs- und Erlebnisräume eingreift (Smulyan, 2000).

\section{Das Gefühl der Isolation}

Als eines der größten mit dem Aufstieg in eine Führungsposition verbundenen Probleme beschrieben die befragten Frauen sowohl in Deutschland als auch in den USA das Gefühl der persönlichen Isolation. Von diesem Problem berichteten sowohl die Frauen, die sich bereits in einer pädagogischen Führungsposition befanden, als auch jüngere Kolleginnen, die sich erst mit dem Gedanken an eine Führungsposition beschäftigten. In beiden Fällen schien der berufliche Aufstieg dazu zu führen, dass man sich aus dem gewohnten Team oder Kollegium entfernte und plötzlich kaum mehr über Ansprechpartner und -partnerinnen mit ähnlicher Interessenlage verfügte. Diese Problematik wurde von allen Frauen als sehr belastend empfunden und kommt in den folgenden Aussagen sehr deutlich zum Ausdruck. So berichtete eine deutsche Schulleiterin:

Das Problem ist, dass man dann mit den Kollegen nicht mehr auf dem gleichen Niveau steht, dass man es also von daher sehr viel schwieriger hat innerhalb des Kollegiums, weil man einfach ganz woanders steht (D Interview 6: 43).

Ganz ähnlich klingen auch die Erfahrungen der amerikanischen Kolleginnen:

I've built up relationships with teachers in my building (...) Even now as I'm studying and people know that I want to be a principal (...) it's like they see you as different, you're not one of the group anymore. And I think when you move down to that office, you're basically isolated from that peer group that you have (US Interview 9: 127-135).

Anytime you cross the line from being a teacher to an aspiring administrator, you are seen as being different: you are not one of us any more, you have the inside track and they don't care what you learnt last month in class and don't want to share (US Interview 10: 84-87). 
Die Entscheidung für eine Führungsposition ist somit nahezu unweigerlich mit einer Neupositionierung innerhalb des bisherigen Arbeitsumfeldes und daher häufig auch mit negativen Auswirkungen auf bestehende soziale Kontakte verbunden. Es drängt sich an dieser Stelle die Frage auf, ob diese Problematik möglicherweise in einem Zusammenhang mit einer spezifisch weiblichen Sozialisation gesehen werden kann, die sich innerhalb einer «Beziehungskultur» vollzieht und an die hohe Wertschätzung freundschaftlicher Beziehungen und sozialer Netzwerke gekoppelt ist (Tannen, 1990). Auf eine solche Problemlage könnten beispielsweise auch in Deutschland gezielte Weiterbildungsangebote für Frauen mit Interesse an einer Führungsposition reagieren (Fahrenwald \& Porter, 2007).

\section{Die Ambivalenz des beruflichen Aufstiegs: Moving up, over, out, down}

Die prinzipielle Ambivalenz des beruflichen Aufstiegs wurde insbesondere in den amerikanischen Interviews immer wieder betont. Selbst eine «erfolgreiche» Karriere wurde hier nicht eindeutig als ein "moving up» beschrieben, sondern auch als ein «moving out» oder «moving down» - erstaunlicherweise jedoch nirgendwo als ein «moving in». Die folgenden Interviewausschnitte verdeutlichen dies exemplarisch:

On a career path it's "moving up" (...) So from that point, from that standpoint, "up" is the right word and along with that "up" comes tremendous accountability and stress and lack of sleep and all these (US Interview 3: 162-164) (Herv. v.d. Verf.).

I guess it's an upward move financially. It's an upward move as far as having more control over your surroundings. It's an upward move as far as gaining respect. But it's a lateral or maybe even a downward move, because maybe you're giving up some of yourself for that. You're definitely giving up more of your time, which means your sacrificing family, hobbies or whatever it is you like to do. You're also giving up action with the students that you have as a teacher. You're giving up good relationships (US Interview 16: 232) (Herv. v.d. Verf.).

[It's] moving out of my classroom, possibly out of my district (...) I think it's more of a lateral move. I mean, obviously you're doing a lot more different things, but the type of position that I want to go into I've already done a lot of those things, so I don't think it is going to be a huge culture shock (...) I feel comfortable in doing all of that (...) But I think the big concern for me, obviously, is the moving out part (US Interview 11: 806, 814) (Herv. v.d. Verf.).

Als ein $A u f s t i e g$ präsentiert sich der berufliche Erfolg somit lediglich im Hinblick auf eine konventionelle Karriererhetorik («on a career path it's moving up»), in 
finanzieller Hinsicht ("I guess it's an upward move financially») oder in Bezug auf den Zuwachs an Einfluss und Respekt («It's an upward move as far as having more control over your surroundings. It's an upward move as far as gaining respect.») Als ein Abstieg oder sogar Ausstieg werden dagegen die mit einer Führungsposition verbundenen Aspekte wie Verantwortung und Stress («tremendous accountability and stress»), Schlafmangel («lack of sleep»), zeitliche Einschränkungen («sacrificing family, hobbies or whatever it is»), der Verlust von Beziehungen ("you're giving up good relationships») sowie die Aufgabe des unmittelbaren Kontakts mit den Schülerinnen und Schülern beklagt («moving out of my classroom»). Es stellt sich somit die Frage, warum sich offensichtlich so viele Frauen in dem gegenwärtig vorherrschenden Karrieremodell so wenig zu Hause fühlen («moving in»).

\section{Ambivalenzen der Macht}

Ein wichtiges Thema stellt in diesem Zusammenhang das Thema der Macht dar. In der Geschlechterhierarchie der Schule gilt Macht traditionell als männlich konnotiert (Kreienbaum, 1995). Bisherige Untersuchungen über Schulleiterinnen im deutschsprachigen Raum bescheinigten diesen häufig auch ein kompliziertes, ja manchmal fast gestörtes Verhältnis zur Macht (von Lutzau, 1996). Diese Tendenz wird auch durch die aktuelle Studie bestätigt - allerdings in dieser Form nur für die deutsche Untersuchungsgruppe.

\section{Macht als negative Instanz: Macht als "Macht über»}

Bei den deutschen Interviewpartnerinnen war eine tendenziell negative Einstellung gegenüber der mit einer Führungsposition verbundenen Ausübung von Macht zu festzustellen. Dies belegen viele ähnliche Aussagen zu diesem Thema:

Normalerweise ist der Begriff der Macht doch sehr negativ. Man denkt nur an die, die Macht über andere ausüben (D Interview 8: 103).

Für mich ist Macht sehr negativ besetzt. Macht ist für mich: Macht, die von Führern ausgeht, von Diktatoren und grundsätzlich eigentlich negativ bestimmt ist. Jemand, der mächtig ist, ist mir von vornherein unsympathisch (D Interview 9: 179).

Macht wird prinzipiell als «Macht über» aufgefasst und ist mit negativen Assoziationen verknüpft («Diktatoren»; «Führer»). Diese Einstellung offenbart sich auch noch in dem Versuch, zwischen einer "positiven" und einer "negativen» Ausübung von Macht zu unterscheiden. So antwortete eine deutsche Schulleiterin auf die Frage, was Macht für sie bedeute: 
Sagen wir mal so: Macht kann ja in positiver und in negativer Weise im Prinzip ausgeübt werden. Wenn ein Schulleiter beispielsweise kraft seines Amtes bestimmte Befugnisse hat, dann hat er die einfach. Natürlich kommt es dann darauf an, wie er sie durchsetzt (D Interview 4: 180).

Ähnlich zurückhaltend formulierte sie daher auch ihren persönlichen Umgang mit Macht:

Ich sehe Macht als zusätzliche PS, die ich unter der Haube habe. Und mit denen ich mich im Notfall noch vor den $L K W$ sozusagen setzen kann. Also, ich fahre sozusagen meinen Wagen nicht ganz aus. Ich drücke nicht voll auf das Gaspedal. Aber ich weiß, dass ich diese PS noch habe. Und dass, wenn ich durchdrücke, dann komme ich in die Zone, wo ich wieder sicher bin (D Interview 1: 291).

Selbst in Situationen, wo die Ausübung von Macht als prinzipiell notwendig erachtet wird, ist somit fast entschuldigend von einem «Notfall» die Rede, der einer besonderen Legitimation bedarf.

\section{Macht als kreative Instanz: Macht als "power»}

Ganz anders klingen die Aussagen im amerikanischen Sample, wo eine eher entspannte Einstellung zum Thema Macht vorherrschte. Macht wurde hier tendenziell als ein Mittel der Gestaltung und Veränderung verstanden und im Sinne von «power» meist positiv konnotiert. Macht wurde hier auch - im Gegensatz zu der in den deutschen Interviews vorherrschenden Auffassung - nicht in erster Linie als "power over», sondern als "power to» interpretiert, d.h. als eine Möglichkeit, etwas zu bewegen und zu gestalten. So lautete die Antwort einer amerikanischen Interviewpartnerin auf die Frage, was Macht für sie bedeute:

Having the authority to instruct teachers to do better and instruct them to do their job according to the law. Not really an actual power, it's more of a guiding power, I would say, rather than an actual power over people (US Interview 18:181).

In eine ganz ähnliche Richtung weisen auch die beiden folgenden Aussagen, die auf die aktive und dynamische Wirkung von Macht abzielen:

I guess, having the power to change things, would be the way that I consider power (US Interview 15: 283).

I think it means being, I guess I should say, being strong and being fair, and really in the end making people work for you (...) I think as an administrator, your power would come from having the ability to make people want to work for you. You know the kids don't want to disappoint you, your teachers want to be great for 
you, they don't want to let you down, they want to help you out, and they want to make you proud (...) So, I think that your power will come, as a principal, just being a "people person", and having people work for you (US Interview 17: 336341).

Macht erscheint in diesen Äußerungen nicht als eine autoritäre, sondern als eine kreative Instanz, die sowohl auf die persönliche als auch auf die organisationale (Weiter-)Entwicklung ausgerichtet ist:

I guess having the power to change things would be the way that I consider power (US Interview 15: 283).

Macht wird auch nicht auf eine bestimmte hierarchische Position bezogen, sondern an die Bereitschaft der einzelnen Mitarbeiterinnen und Mitarbeiter geknüpft, eine Führungspersönlichkeit zu akzeptieren und mit ihr zu kooperieren:

However, if nobody wants to respect you or wanting to give you that kind of position of power or respect or whatever it is, than you're not gonna have it (US Interview 16: 284).

Dieses auf gegenseitigen Respekt und Akzeptanz ausgerichtete Verständnis von Macht impliziert einen zutiefst demokratischen Impuls, der in einem deutlichen Gegensatz zu der in den deutschen Interviews vorherrschenden hierarchischen und autoritären Machtauffassung steht. An dieser Stelle liegt ein Hinweis auf die kulturellen und historischen Unterschiede zwischen Deutschland und den USA nahe: Während sich der deutschsprachige feministische Diskurs in den vergangenen Jahren meist im Anschluss an Foucault mit einer ideologie- und herrschaftskritischen Analyse von Macht beschäftigte, steht das amerikanische Alltagsverständnis von Macht ganz eindeutig in einer Tradition des Pragmatismus und betont die positiven Aspekte von Macht als einem Mittel der Gestaltung. Offen bleiben muss an dieser Stelle die Frage, welcher machttheoretische Zugang einer Erhöhung des Frauenanteils in Führungspositionen auf handlungstheoretischer Ebene zuträglicher sein könnte, und ob nicht gerade auch die aktuelle Schulentwicklungsdebatte in Deutschland von einem solchen pragmatischen Verständnis von Macht profitieren könnte.

Macht als Gestaltungsaufgabe: «Power over» - «power with» - «power to»

In den amerikanischen Interviews wurde Macht häufig auch als "power with» dargestellt, d.h. als eine gemeinsame Gestaltungsaufgabe, die Führungskräfte wie Mitarbeiter gleichermaßen betrifft und prinzipiell auf Teilhabe ausgerichtet ist: 
Certainly the power that the principal has is to (...) is to guide and direct and supervise teachers. Now I think it depends on the type of power that you have is what you go with - is it a power over or a power with... you know, kind of a format (...) Mine is not a power over. Like, I don't tell teachers: "This is what you need to do." I'm more of a power with: Okay, this is what you want. Let's figure out how we're going to do it. You know, I kind of work with them. (US Interview 5: 854-860) (Herv. v.d. Verf.).

You definitely have influence over people (...) And hopefully that it isn't a power over but a power with - a sharing of power. You would hope that everybody could share power - there's enough of it to go around (US Interview 14: 258) (Herv. v.d. Verf.).

In der amerikanischen Schulentwicklungsforschung wurde in diesem $\mathrm{Zu}$ sammenhang das Modell eines «relational leader» entwickelt, das es Schulleitern und Schulleiterinnen ermöglicht, "see[ing] themselves in relationships that are facilitative of others» efforts rather than in control» (Grogan, 1999, S. 524). Im gegenwärtigen Schulalltag in Deutschland herrscht dagegen häufig das Modell eines «instructional leader» vor, bei dem die Führung von Schule und Personal maßgeblich von einer einzelnen Person vollzogen und verantwortet wird (Wissinger, 1996). Dieses traditionelle Modell von Führung erscheint nicht nur aus Sicht der hier befragten Frauen wenig attraktiv, sondern entspricht auch nicht mehr dem aktuellen Stand von Schulentwicklung, bei dem die Schule als ein Gesamtsystem gesehen wird, das von der Zusammenarbeit von Schulleitung, Lehrern, Eltern und Schülern lebt (Altrichter, Schley \& Schratz, 1998).

\section{Die Genderperspektive als Modernisierungs- strategie}

Die Themen «Schulentwicklung» und «Frauenforschung» wurden bislang meist getrennt betrachtet (Koch-Priewe, 1996). Auf diese Weise wurde eine differenzierte Perspektive auf Männer und Frauen in der Schule vernachlässigt (Miller, 2002). Vor dem Hintergrund der aktuellen gesellschaftlichen Modernisierungsprozesse erscheint diese Trennung jedoch nicht länger sinnvoll. Die hier präsentierten Forschungsergebnisse weisen mehrheitlich darauf hin, dass die Unterrepräsentation von Frauen in pädagogischen Führungspositionen nicht lediglich als ein individuelles Problem anzusehen ist, sondern auch unmittelbar mit strukturellen Defiziten im Hinblick auf die traditionellen Begriffe von «Karriere», «Macht» und «Führung» verbunden ist. Diese Begriffe entstammen einem androzentrisch geprägten Denken des 19. und 20. Jahrhunderts, das durch Asymmetrien und Hierarchien gekennzeichnet ist (Klinger, 2000) und sich tief in zentrale Begrifflichkeiten moderner Gesellschaften eingeschrieben hat. In diesem 
Sinne wird der traditionelle Karrierebegriff in der Regel mit Linearität, Durchsetzungsvermögen und strategischem Denken gleichgesetzt und ist eng an die «männliche Normalbiographie» geknüpft. Viele der im Rahmen des hier vorgestellten Projekts befragten Frauen äußerten jedoch ein deutliches Unbehagen an dieser männlich dominierten "Karrierekultur» und verwiesen auf die damit verbundenen Defizite und Probleme. Hier erscheinen die Initiierung einer breit angelegten, öffentlichen Debatte über den überlieferten Karrierebegriff sowie die Entwicklung eines alternativen Verständnisses von «Führung» und «Macht» im Hinblick auf die zukünftige Gestaltung einer geschlechtergerechten Gesellschaft angebracht.

In den Sozialwissenschaften wird seit einigen Jahren die Debatte über eine «Modernisierung der Moderne» (Beck \& Bonß, 2001) geführt. Auf theoretischer Ebene geht es darum, mit Hilfe von Schlüsselbegriffen «Konfliktsemantiken» (Beck, Giddens \& Lash, 1996 S.7) aufzuzeigen, in denen explizite wie implizite Nebenfolgen der traditionellen Moderne aufbrechen und auf die Notwendigkeit verweisen, dass gesellschaftliche Grundüberzeugungen im Großen und Kleinen neu ausgehandelt werden müssen. Die in der vorliegenden Studie über Frauen in pädagogischen Führungspositionen deutlich gewordenen Ambivalenzen vieler Frauen gegenüber dem traditionellen Karrierebegriff legen es nahe, diesen ebenfalls als eine solche "Konfliktsemantik» zu begreifen und aus einer modernisierungstheoretischen Perspektive zu überdenken. Im gegenwärtigen gesellschaftstheoretischen Diskurs gilt Ambivalenz als eine Strukturkategorie der Moderne (Bauman, 1995; Beck, Giddens \& Lash, 1996). Die Kategorie der Ambivalenz wird dabei nicht lediglich als ein Indikator für Unsicherheit und Ungewissheit aufgefasst, sondern auch in ihrem innovativen und kreativen Potential gesehen.

Auf diese Weise eröffnet sich die Chance, tradierte Begrifflichkeiten zu hinterfragen und gegebenenfalls zu revidieren. Eine modernisierungstheoretisch reflektierte Gleichstellungspolitik sollte hier darauf achten, nicht Frauen im Zeichen der Gleichstellung an androzentrische Begrifflichkeiten anzupassen, sondern vielmehr diese Begrifflichkeiten selbst aus einer gendersensiblen Perspektive zu beleuchten und zu re-interpretieren. Am Beispiel der hier behandelten Thematik von Frauen in pädagogischen Führungspositionen könnte dies bedeuten: In Anbetracht der Herausforderungen an ein modernes und geschlechtergerechtes Schulsystem, das auf eine gleichberechtigte Teilhabe von Männern und Frauen in pädagogischen Führungspositionen setzt, erscheint es notwendig und sinnvoll, neue Modelle von Karriere, Führung und Macht zu entwickeln und dabei auch die Erfahrungen und Lebenswirklichkeiten von Frauen zu integrieren. Die Genderperspektive wird auf diese Weise zur Modernisierungsstrategie. 


\section{Literatur}

Altrichter, H., Schley, W. \& Schratz, M. (Hrsg.). (1998). Handbuch zur Schulentwicklung. Innsbruck: Studien-Verlag.

Bastian, A. \& Greer, C. (1985). Choosing equality: The case for democratic schooling. New York: New World Foundation.

Bauman, Z. (1995). Moderne und Ambivalenz. Das Ende der Eindeutigkeit. Frankfurt a.M.: Suhrkamp.

Beck, U., Giddens, A. \& Lash, S. (1996). Reflexive Modernisierung. Eine Kontroverse. Frankfurt a.M.: Suhrkamp.

Beck, U. \& Bonß, W. (Hrsg.). (2001). Die Modernisierung der Moderne. Frankfurt a.M.: Suhrkamp.

Bergmann, N. \& Pimminger, I. (2004). Praxis-Handbuch Gender Mainstreaming. KonzeptUmsetzung - Erfahrung. Wien. Bundesministerium für Wirtschaft und Arbeit.

Biermann, C. \& Koch-Priewe, B. (2004). Gender in der LehrerInnenbildung und Schulentwicklung. In E. Glaser, D. Klika \& A. Prengel (Hrsg.), Handbuch Gender und Erziehungswissenschaft (S. 523-537). Bad Heilbrunn/Obb.: Julius Klinkhardt.

Brehmer, I. (1992). Der widersprüchliche Alltag. Probleme von Frauen im Lehrberuf. Bielefeld: Kleine Verlag.

Chott, P. O. \& Schmirber, G. (Hrsg.). (2003). Konrektorenstudie. Ergebnisse der Befragung von Konrektor(inn)en und Stellvertreter(inn)en an Grund-, Haupt-und Förderschulen in Bayern im Jahr 2002. München: Hanns-Seidel-Stiftung e.V.

Curry, B. K. (2000). Women in Power. Pathways to leadership in education. New York: Teachers College Press.

Fahrenwald, C. \& Porter, M. (2002): Frauen machen Schule. Transatlantisches Forschungsprojekt zu beruflichem Selbstverständnis und Weiterbildungswünschen von Frauen in pädagogischen Führungspositionen, Universität Augsburg (unveröffent. Zwischenbericht).

Fahrenwald, C. \& Porter, M. (2006). Going over to the Dark Side - Ambivalenzerfahrungen in Pädagoginnenkarrieren. Ein transatlantischer Vergleich. In A. Schlüter (Hrsg.), Bildungs- und Karrierewege von Frauen. Wissen - Erfahrungen - biographisches Lernen (S. 202218). Opladen: Verlag Barbara Budrich.

Fahrenwald, C. \& Porter, M. (2007). Weiterbildung als Cultural Change. Transatlantische Studie über Frauen in pädagogischen Führungspositionen. In H. Macha \& C. Fahrenwald (Hrsg.), Gender Mainstreaming und Weiterbildung. Organisationsentwicklung durch Potentialentwicklung (S.131-147). Opladen: Verlag Barbara Budrich.

Flaake, K. (1989). Berufliche Orientierungen von Lehrerinnen und Lehrern. Eine empirische Untersuchung. Frankfurt a.M.: Campus.

Glumpler, E. (1993). Lehrerin - «der» Frauenberuf? Berufsorientierungsprozesse zwischen Abitur und Lehramtsstudium. In E. Glumpler (Hrsg.), Erträge der Frauenforschung für die LehrerInnenbildung (S. 187-221). Bad Heilbrunn: Klinkhardt.

Grogan, M. (1999). Equity/equality issues of gender, race, and class. Educational Administration Quarterly. 35, 4, 518-536.

Hodgkinson, H. \& Montenegro, X. (1999). The U.S. School Superintendent: The invisible $C E O$. Washington, DC: Institute for Educational Leadership.

Kansteiner-Schänzlin, K. (2004). Frauen - Führung - Schule. Eine Sichtung des Forschungsstands zu Schulleiterinnen. Die Schulleitung 2/04, 4-10.

Klinger, C. (2000). Die Ordnung der Geschlechter und die Ambivalenz der Moderne. In: S. Becker, G. Kleinschmidt, I. Nord \& G. Schneider-Ludorff (Hrsg.), Das Geschlecht der Zukunft. Zwischen Frauenemanzipation und Geschlechtervielfalt (S. 29-63). Stuttgart: Kohlhammer.

Koch-Priewe, B. (1996). Frauenförderung durch Organisationsentwicklung an Schulen. In A. Kaiser (Hrsg.), FrauenStärken - ändern Schule (S. 280-186). Bielefeld: Kleine Verlag. 
Kreienbaum, A. (1995). Erfahrungsfeld Schule. Koedukation als Kristallisationspunkt (2. Aufl.). Weinheim: Juventa.

von Lutzau, M. (1996). Wie Schulleiterinnen ihre Rolle sehen und wie sie mit ihrer Macht umgehen. In A. Kaiser (Hrsg.), FrauenStärken - ändern Schule (S. 272-279). Bielefeld: Kleine Verlag.

Miller, S. (2002). Der berufliche Werdegang von Schulleiterinnen - Ausgewählte Ergebnisse einer Untersuchung an Grundschulen in Nordrhein-Westfalen. In J. Wissinger \& S. G. Huber (Hrsg.), Schulleitung - Forschung und Qualifizierung (S. 33-44). Opladen: Leske + Budrich.

Rosenbusch, H. S. (1997). Die Qualifikation pädagogischen Führungspersonals. In E. Glumpler \& H. Rosenbusch (Hrsg.), Perspektiven der universitären Lehrerbildung (S. 147-165). Bad Heilbrunn: Klinkhardt.

Shakeshaft, C. (1993). Gender equity in schools. In C. Capper (Ed.), Educational administration in a pluralistic society (S. 86-109). Albany: Suny Press.

Smulyan, L. (2000). Balancing acts. Women principals at work. New York: State University of New York Press.

Sobehart, H. (Hrsg.) (in press): Sharing the Spirit, Fanning the Flame: Women leading education across the continents. Lantham Maryland, Toronto and Oxford: Rowman and Littlefield.

Tannen, D. (1990). You just don't understand: women and men in conversation. New York: Morrow.

Werle, P. (2001): Zum beruflichen Selbstkonzept des Schulleiters. Eine Untersuchung zum Selbstbild und Selbstverständnis von Schulleiterinnen und Schulleitern sowie deren Bedürfnisse und Erwartungen an die Schulleiterfortbildung, Saarbrücken: Conte Verlag.

Wissinger, J. (1996). Perspektiven schulischen Führungshandelns. Eine Untersuchung über das Selbstverständnis von SchulleiterInnen. Weinheim und München: Juventa.

Schlagworte: Schulentwicklung, Frauen in pädagogischen Führungspositionen, berufliche Identität, Ambivalenzerfahrungen, transatlantischer Vergleich, International vergleichende Erziehungswissenschaft

\section{La perspective des genres en tant que stratégie de modernisation sociétale: une comparaison transatlantique des femmes qui occupent des postes à responsabilité en pédagogie}

\section{Résumé}

Le processus actuel de modernisation sociétale positionne de manière nouvelle la perspective des genres dans les discussions en sciences sociales et politiques. Ainsi en est-il dans les recommandations officielles de la politique européenne prônée par le traité d'Amsterdam de 1998 à propos du Gender Mainstreaming, c'est-àdire de l'égalité entre hommes et femmes dans tous les domaines de la vie. En Allemagne cependant, les femmes occupant des positions dirigeantes se trouvent toujours nettement sous-représentées dans tous les domaines. Ceci est également valable dans le domaine scolaire, un domaine d'application particulièrement sensible pour le développement de la société de demain. Il paraît dès lors pertinent 
et d'intérêt général d'examiner la situation des femmes dans des fonctions de cadres dirigeants en pédagogie au regard des structures traditionnelles du système scolaire. Il s'agit de se focaliser sur cet aspect des recherches sur le management qui, jusqu'à présent, portent très souvent sur l'ensemble des femmes qui occupent des fonctions dirigeantes, mais négligent celles occupant une fonction managériale dans un contexte pédagogique. Qu'est ce qui empêche les femmes de briguer des postes à responsabilité dans le domaine pédagogique ? Quelles expériences ont-elles faites dans ce domaine précisément ? Ces questions sont au centre de la présente contribution qui montre quelques résultats émanant d'un projet de recherche transatlantique. Les pays anglo-américains possèdent une longue tradition de débats à propos des "qualités du leadership». La prise en compte de cette thématique dans un cadre international ouvre d'intéressantes perspectives de coopération et d'analyse comparatives interculturelles.

Mots clés: études de genres, cadres dirigeants, politique européenne en matière d'égalité entre hommes et femmes, comparaisons internationales, gender mainstreaming

\section{La prospettiva Gender come strategia di modernizzazione. Donne in posizioni dirigenti pedagogiche: un confronto transatlantico.}

\section{Riassunto}

Nell'ambito degli attuali processi di modernizzazione della società, è entrata nell'ottica delle discussioni socio-scientifiche e politiche anche una nuova prospettiva Gender. Grazie al Trattato di Amsterdam del 1998 è stato reso possibile il Gender Mainstreaming e con esso la direttiva ufficiale della politica europea per l'applicazione delle pari opportunità uomo-donna in tutti gli ambiti di vita (Bergmann \& Pimminger 2004). In Germania, le donne dirigenti sono però tuttora sottorappresentate in tutti i ruoli sociali importanti. Ciò vale anche per il settore scolastico, un campo d'azione importante per lo sviluppo futuro della società. Sembra perciò sensato e di interesse sociale, prendere in considerazione le donne in posizioni pedagogiche importanti attive nelle strutture scolastiche tradizionali e così dirigere lo sguardo sul campo di ricerca finora poco studiato del gruppo dirigente femminile più trascurato all'interno dell'importante categoria di donne dirigenti (Kansteiner-Schänzlin 2004: 7). Cosa impedisce a una donna di aspirare a una posizione dirigente nel campo pedagogico? Che tipo di esperienze hanno fatto le donne nella loro ascesa per raggiungere tale status? Queste sono domande centrali del contributo che presenta i risultati di un progetto di ricerca transatlantico su questo tema. Nei paesi angloamericani il concetto di "Qualities of Leadership» ha una lunga tradizione. Considerare questa tematica 
nell'ambito internazionale ha permesso di trarne delle prospettive interessanti nell'ottica dei confronti culturali e della cooperazione.

Parole chiave: studi di genere, quadri dirigenti, politica europea in materia di parità di genere, comparazioni internazionali, gender mainstreaming

\section{Gender Perspectives as a Strategy of Modernization: Women in Educational Leadership Positions - A Trans-Atlantic Comparison}

\section{Summary}

A new, greater focus on gender aspects of the modernization process has become de rigueur in social science research as well as in politics. A primary example is the Amsterdam Agreement of 1998 that established «Gender Mainstreaming,» and thereby equalization of the conditions for men and women in all domains, as an officially endorsed principle of European politics (Bergmann \& Pimminger 2004). However, in Germany, women still remain considerably under-represented among leadership positions in all major social spheres. This is also true in regard to school leadership, arguably one of the most significant and influential realms that contributes to future social development. For this reason it makes sense paying greater attention to this «little noticed subset among those women leaders who are gladly heralded as illustrative» Kansteiner-Schänzlin (2004: 7). Therefore it is both good policy as well as of particular social significance to look at the current situation of women who aspire to positions of school leadership in light of the traditional institutional structures that have defined their vocation. Which kinds of issues and concerns arise for women who might aspire to positions of educational leadership? How do both individual as well as institutional constraints color their perceptions and aspirations? What have the experiences been of women who are successfully forging career paths as school administrators? These questions inform the following presentation in which we provide selected results from a Trans-Atlantic research partnership. This research builds on the long tradition of scholarship on "qualities of leadership" that is well-established in English-speaking countries. Therefore, by situating this discussion within the context of an international, comparative study we gain valuable insights into the ways that this discourse has been framed by cultural, linguistic, and policy factors in both the United States and Germany.

Keywords: School policy, women in school leadership positions, identity, ambivalence, comparative education, gender mainstreaming 
T h e m a 